\title{
Better health and wellbeing for a billion more people: integrating non-communicable diseases in primary care
}

\author{
Cherian Varghese and colleagues describe a model to improve equitable access to good quality \\ health services for non-communicable diseases within primary healthcare
}

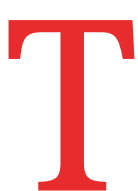
he Alma-Ata declaration of 1978 made the case for comprehensive primary healthcare for all countries. However, the era of the millennium development goals saw an unprecedented increase in financial support for disease specific initiatives (eg, HIV/AIDS, malaria, and tuberculosis) rather than for broader population health or cross cutting programmes, such as improvements in disease prevention, primary care services, and the health workforce. ${ }^{1}$

We examine the evidence supporting the management of non-communicable diseases (NCDs) in primary care and existing models of care, with a focus on the NCDs that cause the most premature mortality (cardiovascular diseases, cancer, chronic respiratory diseases, and diabetes), and propose an approach for the management of NCDs in settings with limited resources.

\section{Role of primary care in controlling non- communicable diseases}

Primary care is more than a first point of care; it is the core process of a health system. It is accessible to all patients and can undertake the management of early stages of NCDs by providing first contact, continuity, and integration of care. Primary care becomes an effective way to manage NCDs

\section{KEY MESSAGES}

- The increasing burden of NCDs is overwhelming health systems resulting in high costs to governments and individuals

- Integrating NCDs in primary care is a cost effective, affordable, and equitable model of care that can reduce morbidity and mortality from NCDs

- More evidence on NCD management in primary care and greater advocacy for integration of NCDs in primary care are needed when it moves from delivering an episode of care to providing an integrated approach that includes prevention, diagnosis, treatment, and palliative care for all conditions and over time. ${ }^{2}$

In well developed health systems most of the care for NCDs is managed by primary care; however, most primary care facilities in poorer settings are not equipped to detect and treat NCDs. Surveys by the World Health Organization on country capacity report that only $6 \%$ of low income countries compared with $85 \%$ of high income countries have the necessary equipment generally available (that is, available in $50 \%$ or more public health sector facilities) to take six essential primary care measurements-height, weight, blood glucose, blood pressure, total cholesterol, and urine albumin. ${ }^{3}$

\section{What the evidence shows}

We reviewed evidence on the effect of integrating and prioritising the management of NCDs in primary care on mortality, quality of care, and cost effectiveness.

\section{Mortality reduction}

Evidence from New Zealand, Mexico, countries of the Organisation for Economic Cooperation and Development countries, and England and Wales shows that reduction in cause specific premature mortality from asthma and bronchitis or emphysema, cancer, and cardiovascular disease is attributed to treatment and improvements in primary care. ${ }^{4-7}$

\section{Priorities in poor settings}

A systematic review of primary care models for NCD interventions in sub-Saharan Africa concluded that screening patients when they attend health services is suitable in poor settings, rather than more costly population screening programmes. It also identified the most important needs for NCD management in primary care, including essential diagnostic tools and medications, standardised procedures, and referral support. ${ }^{8}$ Every consultation can be used as an opportunity to promote healthy lifestyles and detect conditions early or before symptoms appear. The WHO package of essential non-communicable disease interventions (WHO-PEN) for primary healthcare, which has been adapted in about 30 countries, is a set of cost effective and action oriented interventions feasible in all settings. ${ }^{9}$ The essential packages on cardiovascular, respiratory, and related disorders, and cancer of the Disease Control Priorities Network are another set of interventions that can be used for integration in primary care ${ }^{1} 0$

\section{Quality of care}

Improvement in the quality of primary care services in Brazil, especially in poor and vulnerable communities, led to a reduction in hospital admissions. ${ }^{11}$ Interventions to improve quality of care range from policies and regulations, mixed payment methods (eg, capitation with a set fee per patient and incentives for performance designed to motivate and encourage care providers), improved capacity of health information systems, and improvements in the capacity and quality of management within primary care facilities. Primary care increases access to healthcare services, improves health outcomes and quality of life for patients with NCDs, and decreases hospital admissions. ${ }^{12}$

\section{Cost effectiveness}

A well functioning primary care system, which includes ambulatory primary care delivered through local hospitals, is cost effective and can manage up to $90 \%$ of healthcare demand in low and middle income countries. ${ }^{10}$ Task shifting, which uses trained non-physician healthcare workers to do tasks traditionally done by doctors according to the local context, accompanied by appropriate health system support is a cost effective strategy. ${ }^{13}$ The economic cost of drug therapy and counselling for people at high risk of cardiovascular diseases is 0.29 international dollar per million population a year for low and 
middle income countries (international dollar is a hypothetical unit of currency with the same purchasing power as US\$ in the United States at a given point in time). ${ }^{14}$ Ethiopia reported that hypertension management alone is an effective intervention to prevent poverty. ${ }^{15}$

\section{Examples from countries}

Brazil's health system started in 1996 and is based on decentralised universal access with municipalities providing comprehensive and free healthcare. Today, 27000 family health teams are active in nearly all Brazil's municipalities, each serving up to about 2000 families or 10000 people, and annual resources for primary healthcare have increased. ${ }^{16}$

In Thailand, a primary care system based on community participation started in 1985 through the recruitment of health volunteers and the establishment of health centres and a drug cooperative system. Outof-pocket payment dropped from $44.5 \%$ in 1994 to $12.4 \%$ in 2011 because of full implementation of the universal coverage scheme in 2002. ${ }^{17}$

It is encouraging to see that Kerala state in India is transforming primary healthcare centres into family health centres with a defined set of services and the necessary support elements. ${ }^{18}$

Model for management of NCDs in primary care The challenge in systems focused on maternal and child health and communicable diseases is to integrate and scale up services for NCDs. We propose a model for NCD management in primary care (box 1) to prevent, detect, and manage NCDs at an earlier stage. This is based on the systematic review of primary care models for NCDs in sub-Saharan Africa by Kane et al. ${ }^{8}$

To adequately respond to the needs of people with NCDs and enable proactive population management, primary care in many settings will need to change to include the following:

- Multidisciplinary teams with diverse competencies (eg, health education, dietary education, medication management, and social care);

- The right to prescribe medicines for trained midlevel non-physician care providers

- Availability of essential diagnostic tools and medications

- Improved health information systems that use unique patient identifiers

- Family and community based models of care (including collaboration with public health officials to tackle local determinants of health)
- Better mechanisms for referral and counter referral.

When relevant, NCD care will need be integrated into existing programmes for tuberculosis, HIV, and maternal health services.

Primary care services should be tailored to a defined catchment population to enable continuity of care and responsiveness to the changing disease burden. The size of the catchment population for primary care can be determined by the disease burden, population density, health workforce model, and available resources. Transforming the current model of care to make primary care the main provider of care for NCDs will require more efficient use of existing resources and, in many cases, additional resources for health services, with an increase in infrastructure, medical products, health workers, health information systems, and managerial capacity directed to primary care. Appropriate policy changes are needed for medical education and professional regulation to strengthen and expand primary care.

\section{Next steps}

Evidence on NCDs mostly comes from high income countries. An analysis of 797 Cochrane reviews with 8850 trials reported that only $13(0.15 \%)$ were done in low income countries. ${ }^{19}$ More research on relevant populations and interventions is needed to provide better evidence and demonstrable outcomes. Scaling up coverage of evidence based interventions for NCDs in primary care could play a major part in reaching the target of the sustainable development goals to reduce premature mortality from NCDS by one third by 2030 through prevention and treatment.

In addition to achieving cure where possible, the goals of NCD care are to improve functional status, prolong and improve quality of life, and provide palliative care. Countries should invest in primary care, and the models of care in each context need to explicitly tackle the contextual challenges and tailor the care to improve outcomes from NCDs. Strong and sustained advocacy is needed at different management and policy levels to

Box 1: Elements of a model for managing non-communicable disease (NCDs) in primary care

Prevention: modify risk factors and promote health

- Tobacco cessation

- Dietary advice

- Reduction in salt intake

- Reduction in alcohol intake

- Promotion of physical activity

- Body weight control

- Counselling

Screening/case finding

- Targeted screening (using factors such age group, tobacco use, obesity) to detect people with NCDs

\section{Management}

Health services

- Decentralised care

- Local clinical guidelines

- Essential diagnostics

- Essential medicines

- Systematic monitoring and evaluation

Human resources

- Adequate staffing

- Continued capacity building

- Competency for NCD services ensured

Decision support

- Adherence to clinical guidelines

- Adherence to medications

- Adherence to follow up

- Effective communication

Quality improvement

- Supportive supervision and mentoring

- Monitoring of quality indicators

- Implementation research 
change the perception that hospitals and subspecialised health facilities are the best way to improve healthcare for NCDs. Public education about the benefits of primary care as well as strengthening of the services provided can build people's trust in primary care for the management of NCDs.

For other articles in the series see www.bmj.com/ NCD-solutions

Contributors and sources:CV conceived the paper, and $C V$ and BN wrote the first draft. MM and IO conducted the literature review, and SB and TC contributed to the content and concepts. All authors revised the manuscript for important intellectual content and approved the final version. CV is the guarantor. CV has experience on NCD management in different countries and helps develop guidance on this topic. BN has extensive experience in designing and evaluating primary care and in teaching community medicine. MM and 10 work in evidence review including on primary care. SB works in primary healthcare and TC in partnerships and coordination for NCD prevention and control in WHO. The views expressed in the document are those of the authors and do not represent the views of the institutions they are affiliated to.

Competing interests: We have read and understood BMJ policy on declaration of interests and have no relevant interests to declare.

Provenance and peer review: Commissioned; externally peer reviewed.

This article is part of a series proposed by the WHO Global Coordination Mechanism on NCDs and commissioned by The BMJ, which peer reviewed, edited, and made the decision to publish. Open access fees are funded by the Swiss Agency for Development and Cooperation, International Federation of Pharmaceutical Manufacturers and Associations (IFPMA), UNOPS Defeat-NCD Partnership, Government of the Russian Federation, and WHO.

Cherian Varghese, coordinator, management of NCDs ${ }^{1}$ Baridalyne Nongkynrih, professor ${ }^{2}$ Igho Onakpoya, senior research fellow ${ }^{3}$ Marcy McCall, senior research fellow ${ }^{3}$ Shannon Barkley, technical officer, primary healthcare services ${ }^{1}$

Téa E Collins, adviser, global coordination mechanism on NCDs ${ }^{1}$
${ }^{1}$ World Health Organization, Geneva, Switzerland ${ }^{2}$ Centre for Community Medicine, All India Institute of Medical Sciences, New Delhi, India

${ }^{3}$ Nuffield Department of Primary Care Health Sciences, University of Oxford, Oxford, United Kingdom

Correspondence to: CVarghese varghesec@who.int

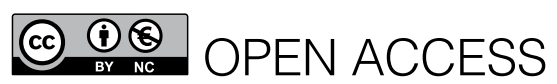

This is an Open Access article distributed under the terms of the Creative Commons Attribution IGO License (https://creativecommons.org/licenses/ by/3.0/igo/), which permits use, distribution, and reproduction in any medium, provided the original work is properly cited.

\section{Check for updates}

1 De Maeseneer J, van Weel C, Egilman D, Mfenyana K, Kaufman A, Sewankambo N. Strengthening primary care: addressing the disparity between vertical and horizontal investment. BrJ Gen Pract 2008;58:3-4. doi:10.3399/bjgp08X263721

2 FrenkJ. Reinventing primary health care: the need for systems integration. Lancet 2009;374:170-3. doi:10.1016/S0140-6736(09)60693-0

3 World Health Organization. Assessing national capacity for the prevention and control of NCDs. 2017. http://www.who.int/ncds/surveillance/ncdcapacity/en/

4 Capewell S, Beaglehole R, Seddon M, McMurray Explanation for the decline in coronary heart disease mortality rates in Auckland, New Zealand, between 1982 and 1993. Circulation 2000;102:1511-6. doi:10.1161/01.CIR.102.13.1511

5 Borja-Aburto VH, González-Anaya JA, Dávila-Torres J, Rascón-Pacheco RA, González-León M. Evaluation of the impact on non-communicable chronic diseases of a major integrated primary health care program in Mexico. Fam Pract 2016;33:219-25. doi:10.1093/ fampra/cmv049

6 Macinko J, Starfield B, Shi L. The contribution of primary care systems to health outcomes within Organization for Economic Cooperation and Development (OECD) countries, 1970-1998. Health Serv Res 2003;38:831-65. doi:10.1111/14756773.00149

7 Unal B, Critchley JA, Capewell S. Explaining the decline in coronary heart disease mortality in England and Wales between 1981 and 2000. Circulation 2004;109:1101-7. doi:10.1161/01. CIR.0000118498.35499.B2
8 Kane J, Landes M, Carroll C, Nolen A, Sodhi S. A systematic review of primary care models for noncommunicable disease interventions in Sub-Saharan Africa. BMC Fam Pract 2017;18:46. doi:10.1186/ s12875-017-0613-5

9 World Health Organization. Tools for implementing WHO PEN (Package of essential noncommunicable disease interventions). 2018. http://www.who.int/ ncds/management/pen_tools/en/

10 Prabhakaran D, Anand S, Gaziano TA, Mbanya J-C, Wu Y, Nugent R. Cardiovascular, respiratory, and related disorders. Disease control priorities. 3rd ed. Volume 5. World Bank; 2017. https://openknowledge. worldbank.org/handle/10986/28875

11 Fausto MCR, Bousquat A, Lima JG, et al. Evaluation of Brazilian primary health care from the perspective of the users: accessible, continuous, and acceptable?) Ambul Care Manage 2017;40(Suppl 2 Supplement, The Brazilian National Program for Improving Primary Care Access and Quality (PMAQ)):S60-70. doi:10.1097/JAC.0000000000000183

12 Shi L. The impact of primary care: a focused review. Scientifica 2012, doi:10.6064/2012/432892

13 Joshi R, Alim M, Kengne AP, et al. Task shifting for non-communicable disease management in low and middle income countries-a systematic review. PLoS One 2014;9:e103754. doi:10.1371/journal. pone.0103754.

14 World Health Organization. Technical annex. Updated Appendix 3 of the WHO Global NCD Action Plan 2013-2020. 2017. http://www.who.int/ncds/ governance/technical_annex.pdf?ua=1

15 Verguet S, Olson ZD, Babigumira JB, et al. Health gains and financial risk protection afforded by public financing of selected interventions in Ethiopia: an extended cost-effectiveness analysis. Lancet Glob Health 2015;3:e288-96. doi:10.1016/S2214109X(14)70346-8

16 Flawed but fair: Brazil's health system reaches out to the poor. 2008. https://www.who.int/bulletin/ volumes/86/4/08-030408/en/.

17 World Health Organization. Primary health care systems (PRIMASYS). Case study from Thailand., 2017. http://www.who.int/alliance-hpsr/projects/ alliancehpsr thailandabridgedprimasys.pdf

18 Government of Kerala. Health and family welfare department. 2017. http://dhs.kerala.gov.in/docs/ transfer/Judgements/aardram_100817.pdf.

19 Heneghan C, Blacklock C, Perera R, et al. Evidence for non-communicable diseases: analysis of Cochrane reviews and randomised trials by World Bank classification. BMJ Open 2013;3:e003298. doi:10.1136/bmjopen-2013-003298.

Cite this as: BM/ 2019;364:kl327

http://dx.doi.org/10.1136/bmj.l327 